\title{
METRIC INEQUALITIES AND THE ZONOID PROBLEM
}

\author{
H. S. WITSENHAUSEN
}

Abstract. For normed spaces the hypermetric and quasihypermetric properties are equivalent and imply the quadrilateral property. The unit ball of a Minkowski space is a zonoid if and only if the dual space is hypermetric. The unit ball of $l_{p}^{n}$ is not a zonoid for $n=3, p<\log 3 / \log 2$, and for $p \leqq 2-(2 n \log 2)^{-1}+o\left(n^{-1}\right)$. The elliptic spaces $\mathscr{E}^{d}, d>1$, are not quasihypermetric.

A metric space $(S, d)$ is said to be hypermetric (Kelly [3]) when

$$
\sum_{i, j=1}^{n} w_{i} w_{j} d\left(x_{i}, x_{j}\right) \leqq 0
$$

for all $n>0, x_{1}, \cdots, x_{n}$ in $S$, and $w_{1}, \cdots, w_{n}$ integers with sum 1 . This implies [5] that (1) also holds for real $w_{i}$ of sum 0 , which is called the quasihypermetric property.

A piecenise linear inequality (PLI) is a relation of the form

$$
\sum_{i=1}^{k} c_{i}\left|\sum_{j=1}^{n} a_{i j} x_{j}\right| \geqq 0
$$

which holds for all $n$-tuples $x_{1}, \cdots, x_{n}$ of real numbers, with fixed real $c_{i}$ and $a_{i j}$. An example is the quadrilateral inequality [8]

(3) $|x|+|y|+|z|-|x+y|-|y+z|-|z+x|+|x+y+z| \geqq 0$.

Since the real line is hypermetric [4], (1) generates an infinite family of PLI's of the form

$$
\sum_{i, j=1}^{n}\left(-w_{i} w_{j}\right)\left|x_{i}-x_{j}\right| \geqq 0
$$

for $w_{i}$ integers of sum 1 and for $w_{i}$ reals of sum 0.

The PLI (2) is said to extend to the normed space $N$ if it holds with the absolute value function replaced by the norm and $x_{1}, \cdots, x_{n}$ arbitrary elements of $N$.

Received by the editors November 13, 1972 and, in revised form, January 23, 1973.

AMS (MOS) subject classifications (1970). Primary 42A40; Secondary 50C10, 52A20.

Key words and phrases. Metric inequalities, zonoids, hypermetric spaces, elliptic geometry.

(c) American Mathematical Society 1973 
A zonoid [1], [2] is a convex body belonging to the closure (in the Hausdorff set metric) of the class of zonotopes (polytopes which are Minkowski sums of segments).

The theorems of I. J. Schoenberg [7] and P. Lévy [6] imply that the above concepts are related.

Proposition 1. For a real normed space $N$ the following 3 properties are equivalent.

(i) every PLI extends to $N$,

(ii) $N$ is quasihypermetric,

(iii) $e^{-\|x\|}$ is positive definite on $N$.

Proof. If every PLI extends to $N$ then in particular $N$ is quasihypermetric. Following an argument of Schoenberg, consider $n+1$ points $x_{0}, \cdots, x_{n}$ in $N$ with weights $-\sum_{i=1}^{n} w_{i}, w_{1}, \cdots, w_{n}$ where the $w_{i}$ are arbitrary reals. This yields

$$
\sum_{i, j=1}^{n} w_{i} w_{j}\left(\left\|x_{i}-x_{0}\right\|+\left\|x_{j}-x_{0}\right\|-\left\|x_{i}-x_{j}\right\|\right) \geqq 0,
$$

that is, the parenthesis is positive definite. Then its exponential is positive definite and, absorbing $e^{\left\|x_{i}-x_{0}\right\|}$ into $w_{i}, e^{-\|x\|}$ is shown to be positive definite. Conversely, if $e^{-\|x\|}$ is positive definite on $N$ it is positive definite on every finite dimensional subspace of $N$. By Lévy's theorem [6], [1] these subspaces are isometrically isomorphic to subspaces of $L_{1}(0,1)$ to which any PLI extends by integration. Since each PLI involves only finite systems of vectors, it extends to all of $N$.

In [4] Kelly raised the question of the possible relations between the hypermetric and quadrilateral properties in normed spaces. Applying Proposition 1 one has

COROLLARY 1.1. For realnormed spaces, the hypermetric and quasihypermetric properties are equivalent and they imply the quadrilateral property.

For $1 \leqq p \leqq 2, e^{-\|x\|}$ is known [7] to be positive definite on $L_{p}(0,1)$, hence

COROLlaRY 1.2. $L_{p}(0,1)$ (and a fortiori $l_{p}^{n}$ ) is hypermetric and quadrilateral for $1 \leqq p \leqq 2$.

This had been conjectured by Kelly [4] and the Smileys [8]. For finite dimensional real normed spaces (Minkowski spaces) the positive definiteness of $e^{-\|x\|}$ is equivalent [1] to the property that the unit ball of the dual space is a zonoid. Thus one has

COROLlary 1.3. The unit ball of a Minkowski space is a zonoid if and and only if the dual space is hypermetric. 
Thus the known fact that all Minkowski planes are hypermetric [4] follows from the elementary fact that all centrally symmetric convex polygons are sums of segments.

For $n \geqq 3$, let $p_{n}$ be the smallest $p$ such that the unit ball of $l_{p}^{n}$ is a zonoid. One has $p_{3} \leqq p_{n} \leqq p_{n+1} \leqq 2$, Bolker [1], [2] has conjectured that $p_{3}=2$. He reports the following bounds of Rosenthal: $p_{3}>\log 9 / \log 7$ and $p_{n}>$ $2 \log n / \log 3 n$, hence $p_{n} \geqq 2-\log 9 / \log n+o\left((\log n)^{-1}\right){ }^{1}{ }^{1}$ These bounds can be substantially improved.

Proposition 2. One has $p_{3} \geqq \log 3 / \log 2$ and $p_{n} \geqq 2-1 / 2 n \log 2+o\left(n^{-1}\right)$.

Proof. For $n=3, p<\log 3 / \log 2$ the quadrilateral inequality in the dual space is violated for $x=(1,1,-1), y=(1,-1,1), z=(-1,1,1)$, as observed by the Smileys [8]. ${ }^{2}$ For large even $n=2 m$, consider the quasihypermetric inequality in the dual $l_{q}^{2 m}$, with $w_{i}=1$ at the $2^{m}$ points with the first $m$ coordinates equal to \pm 1 and the last $m$ coordinates $0, w_{i}=-1$ at the $2^{m}$ points with first $m$ coordinates 0 and the last $m$ equal to \pm 1 . All distances between the two sets are $(2 m)^{1 / q}$ while distances within each set are of the form $2 k^{1 / a}$ with $0 \leqq k \leqq m$. Counting the number of occurrences of each distance, a violation of the inequality is seen to require

$$
2\left(2^{m-1} \sum_{k=0}^{m}\left(\begin{array}{l}
m \\
k
\end{array}\right)\left(2 k^{1 / q}\right)\right)>2^{2 m}(2 m)^{1 / q}
$$

or $2 E\left\{k^{1 / q}\right\}>(2 m)^{1 / q}$ with $k$ binomially distributed. For large $m$, expand $k^{1 / q}$ about the mean $k=m / 2$ and let $1 / q=\frac{1}{2}+\varepsilon$. Then the violation occurs for $\varepsilon<-(16 m \log 2)^{-1}+o\left(m^{-1}\right)$, so that $p_{n} \geqq 2-(2 n \log 2)^{-1}+o\left(n^{-1}\right)$ as claimed.

Kelly [3] has shown that spherical spaces are hypermetric. This no longer holds when antipodes are identified.

Proposition 3. The elliptic plane $\mathscr{E}^{2}$ is not quasihypermetric.

Proof. Assume the opposite, and consider the function, defined for $\mu$ in $C\left(\mathscr{E}^{2}\right)^{*}$, by $F(\mu)=\int \mu(d x) \int \mu(d y) \overline{x y}$, where $\overline{x y}$ is the elliptic distance and the integrals range over the compact space $\mathscr{E}^{2}$. By (1) the function is nonpositive, hence concave on the subspace $\left\{\mu \mid \int \mu(d x)=0\right\}$. The concavity holds as well on the parallel subspace $\left\{\mu \mid \int \mu(d x)=1\right\}$ and in particular on the set $\mathscr{P}$ of probability measures on $\mathscr{E}^{2}$. For $\mu$ in $\mathscr{P}$ and $\tau$ in the compact group $G$ of isometries of $\mathscr{E}^{2}$, let $\mu^{*}$ be the mixture of the displaced measures $\mu \circ \tau$ under normalized Haar measure on $G$. Then $\mu^{*}$ is the uniform.

\footnotetext{
${ }^{1}$ Thus $L_{p}(0,1)$ is not hypermetric for $p>2$.

${ }^{2}$ Alternatively, the hypermetric inequality is violated for the choice of $w_{i}^{\prime}=1$ at $( \pm 1, \pm 1,0)$ and $w_{i}=-1$ at $(0,0,0),(0,0, \pm 1)$.
} 
distribution on $\mathscr{E}^{2}$ and by concavity $F\left(\mu^{*}\right) \geqq F(\mu)$. However, the distribution $\mu$ assigning equal probabilities to the vertices of an equilateral triangle of side length $D$, the diameter of $\mathscr{E}^{2}$, yields $F(\mu)=2 D / 3$ while $F\left(\mu^{*}\right)=2 D / \pi$, a contradiction.

That $\mathscr{E}^{2}$ is not hypermetric already follows from the violation of the hypermetric inequality that occurs for the choice of $w_{i}=-1$ at 3 mutually orthogonal lines and $w_{i}=+1$ at their 4 trisectors.

Since $\mathscr{E}^{2} \subset \mathscr{E}^{d}$ for $d>2$ one has

COROLlaRY 3.1. For $d>1$ the elliptic space $\mathscr{E}^{d}$ is not quasihypermetric.

\section{REFERENCES}

1. E. D. Bolker, A class of convex bodies, Trans. Amer. Math. Soc. 145 (1969), 323-345. MR 41 \#921.

2. - The zonoid problem, Amer. Math. Monthly 78 (1971), 529-531.

3. J. B. Kelly, Combinatorial inequalities, Combinatorial Structures and Their Applications, Gordon and Breach, New York, 1970, pp. 201-207.

4. - Metric inequalities and symmetric differences, Inequalities-II (Proc. Second Sympos., U.S. Air Force Acad., Colo., 1967), Academic Press, New York, 1970, pp. 193-212. MR 41 \#9192.

5. - Hypermetric spaces and metric transforms, Inequalities-III, Academic Press, New York, 1972, pp. 149-158.

6. P. Lévy, Théorie de l'addition des variables aléatoires, Gauthier-Villars, Paris, 1937.

7. I. J. Schoenberg, Metric spaces and positive definite functions, Trans. Amer. Math. Soc. 44 (1938), 522-536.

8. D. M. Smiley and M. F. Smiley, The polygonal inequalities, Amer. Math. Monthly 71 (1964), 755-760. MR 30 \#1384.

Bell Telephone laboratories, InC., Murray Hill, New Jersey 07974 